\title{
The Role of Suboptimal Home-Measured Blood Pressure Control for Cognitive Decline
}

\author{
Teodora Yaneva-Sirakova ${ }^{a}$ Rumiana Tarnovska-Kadreva ${ }^{a}$ \\ Latchezar Traykov ${ }^{\mathrm{b}}$ \\ ${ }^{a}$ Cardiology Clinic and ${ }^{b}$ Neurology Clinic, University Hospital 'Alexandrovska', Sofia, Bulgaria
}

\section{Key Words}

Home-measured blood pressure - Neuropsychological tests $\cdot$ Cognitive impairment •

Vascular risk factors

\begin{abstract}
Aim: We aim to analyze if there is any correlation between suboptimal home-/self-measured blood pressure values and the results from neuropsychological screening tests for early cognitive impairment. Methods: We studied 325 patients with treated hypertension. Mean age was 66.12 ( \pm 10.1 ) years. There were 119 (36.6\%) male and 206 (63.4\%) female patients, among them $52(16 \%)$ with atrial fibrillation. Neuropsychological tests performed were the Mini-Mental State Examination, Montreal Cognitive Assessment, and Hachinski Ischemic Score; additionally, home-measured blood pressure was used. Results: There is a nonlinear age- and risk factordependent correlation between early stages of cognitive impairment and suboptimal homemeasured blood pressure. Conclusion: The use of specific and sensitive neuropsychological tests for early cognitive impairment in patients with suboptimal home-measured blood pressure is effective in the everyday practice.

Copyright $\odot 2012$ S. Karger AG, Basel
\end{abstract}

Cognitive impairment is an emerging social problem. According to the World Health Report of the WHO (World Health Organization), it is a significant part of the health burden for modern populations with approximately 29 million registered to suffer from dementia, of which Alzheimer's disease is predominant and vascular cognitive impairment is second 
in frequency. There is an exponential growth of the number of cases above the age of 60 years, and the world's morbidity is expected to reach 80 million by the year 2025 [1]. As some national recommendations advice, we should suspect cognitive impairment only if there is a history of self-reported decline or a decline observed by relatives or friends in occupational, social, or day-to-day functional status [2]. But at the time when a person or his/her relatives or caregiver suspects some stage of cognitive impairment on the basis of impaired daily living or communication, it will be too late for primary prevention. There are two reasons for this: the first is the continually rising age of the population and the second is the high frequency of cognitive impairment progression towards dementia [3, 4]. The idea of the 'brain at risk', i.e. the mere presentation of cardio-vascular risk factors, comes in accordance with the need for timely prophylaxis [5]. The strength and direction of a correlation between vascular risk factors, especially hypertension and cognitive impairment, is a matter of longstanding debate [6]. Hypertension is the leading risk factor for dementia. On the one hand, hypertension is a major health problem both in the young and in the elderly and, on the other hand, the risk for developing hypertension in the 60-year-olds during their remaining life according to the Framingham study is $90 \%$ [7]. Even though $87 \%$ of the patients are on combination therapy, target office blood pressure (BP) control $(<140 / 90 \mathrm{~mm} \mathrm{Hg})$ is achieved in $27.1 \%$ only, the corresponding control rate for ambulatory $\mathrm{BP}(<130 / 80 \mathrm{~mm} \mathrm{Hg})$ being $35.7 \%$ [8].

There are conflicting data on the correlation between BP and cognitive impairment. In 2005, Cacciatore et al. [9] proposed a U-shaped curve for the cognitive impairment mortality rate, i.e., very high and very low diastolic BP values were associated with an added relative mortality risk. The hypothesis of a U-shaped curve was revived in the results from the ACCORD trial [10], but what is exactly the case with cognitive impairment is an unresolved problem, as are the target values for cognitive impairment protection. The correlation between BP and cognitive impairment is much more complicated than expected and surely not linear as we would prefer it to be.

Our team recognizes the need for effective screening for early cognitive impairment in a population with cardiovascular risk factors. We use the term 'early cognitive impairment' as a collective one for all those cases with mild cognitive impairment (at risk for progression towards Alzheimer's disease), for vascular cognitive impairment/no dementia (at elevated risk for vascular dementia development), and for patients with clinical signs that may lead to progression to mixed dementia. There are sufficient data to suggest that hypertension and its variables are major risk factors for both vascular dementia and Alzheimer's disease. So, we do not focus on the specific type of early cognitive impairment but rather try to establish the need for early cognitive impairment screening in certain risk groups without clinical evidence for developed dementia and impaired daily functioning. Our aim is to define the patients at risk and to emphasize the need for prevention from progression towards dementia.

We are in search of easily administered, time-saving, cost-effective and yet sensitive and specific enough screening tools for early cognitive impairment detection in the everyday clinical practice. Here, the term early cognitive impairment has been used throughout. The reason for this is that during our screening, we assessed and screened for both mild cognitive impairment and early vascular cognitive impairment, as well as mixed-type cognitive impairment. There are sufficient data to support the understanding of a significant contribution of vascular risk factors (mainly hypertension or hypotension) to the pathogenesis and progression to both Alzheimer's disease and vascular dementia, as well as mixed-type dementia.

The main purposes of this preliminary screening stage of the study are:

(1) Is there cognitive impairment in treated hypertensive people with suboptimal BP control? 
(2) Are there other factors that affect cognitive impairment in combination with hypertension?

(3) Is there any early screening tool that is effective, specific, and sensitive enough to raise any suspicion for early cognitive impairment in patients with risk factors?

(4) What is the role of home-measured BP in early cognitive impairment detection?

\section{Patients and Methods}

We used specific and sensitive neuropsychological tests for screening of treated hypertensive patients for cognitive impairment. The population consisted of patients with a history for arterial hypertension. The majority of the patients were on combination therapy at the time of the screening. For every patient, we acquired full medical history, especially hypertension history (including duration, treatment, treatment duration, maximal values, usually measured values, frequency of hypertensive crises, familial history of hypertension, factors that may lead to secondary hypertension, and hypotension crises). All of the patients had a thorough physical examination and basic laboratory screening. Recent data suggest that homemeasured BP might be more reliable in the detection of elevated BP and poor control than office $\mathrm{BP}$, as it provides a more precise initial diagnosis and a more accurate titration of drug treatment. There are relatively few results using this type of measurement, although homemeasured BP is very important. Detection of elevated BP during self-measurement is the most common incentive to seek medical consultation. Hypertension treatment is tailored to selfmeasured values, and this is the method which enables the patient to assess the success of treatment and in turn search medical support or not. Above all, home-measured BP is a relatively precise method, provided that patients are educated how to do it properly. These are the reasons why we decided to utilize home-measured BP in our study rather than office BP. Patients were instructed how to measure their BP properly and in accordance with the European recommendations, and the use of validated upper-arm monitoring devices was strongly recommended [12]. The patients were asked to report the values of their self-measured BP during the following visits. They should not have smoked and should not have had a meal or performed physical exercises 30 min before measurement. They were instructed to first sit comfortably for at least $5 \mathrm{~min}$, with legs uncrossed and at normal temperature, with their back supported. The arm at which the measurement is to be taken should be supported at heart level. Only validated semi-automated or automated oscillometric (electronic) arm cuff devices were recommended to patients. Measurement should be taken with proper cuff size. Initial measurements included two measurements in the morning (before drugs intake) and two in the evening (before eating) every day for a week. The threshold level is $135 / 85 \mathrm{~mm} \mathrm{Hg}$ [13].

We used a battery of neuropsychological tests to assess cognitive function in hypertensive patients, namely the Mini-Mental State Examination (MMSE) and the Montreal Cognitive Assessment (MoCA). The MMSE is a universally accepted neuropsychological tool that is used as a gold standard in the majority of the dementia trials. It is a validated and powerful tool for diagnosis in the advanced stages of cognitive impairment, but quite insensitive to the subtle changes in executive functioning in early/mild cognitive impairment. Cognitive areas tested with MMSE are: orientation, registration, attention, calculation, recall, language, repetition, memory, three-stage command, reading, writing, visual-spatial functions, and drawing/copying. A cutoff threshold of 24 points was used to define cognitive impairment. The lower sensitivity for early/mild cognitive impairment of the MMSE prompted us to include another, more specific test for these particular stages, namely the MoCA. The MoCA was translated into Bulgarian for the first time by our team and published on the official MoCA site. It was used in this study with the kind permission of its creator. This is a 10-min 
test with a maximal score of 30 points and a cutoff threshold of 26 points. It was developed as a compilation of neuropsychological tests with emphasis on frontal executive functioning and attention. This is the reason for its sensitivity for non-Alzheimer dementia. In patients with an MMSE score between 24 and 30, in whom there is still suspicion for cognitive impairment, MoCA is a powerful tool for its detection [14, 15]. Cognitive domains that are focused on in MoCA are: attention and concentration, executive functions, memory, language, visuo-constructional skills, conceptual thinking, calculations, and orientation. MoCA is a kind of neuropsychological tests compilation: alternating trial making test (modification of B-variant), cube-drawing and clock-drawing (visuo-constructional skills), naming, memory, forward digit span, backward digit span, vigilance, serial seven-s (attention testing), sentence-repetition, verbal fluency adapted for Bulgarian language (letter $\mathrm{M}$ used), abstraction, delayed recall (along with cues to differ retrieval from encoding deficits), and orientation. Both neuropsychological tests (MoCA and MMSE) were validated for the Bulgarian population, and a fairly good correspondence between the test results and the clinical state was found. The tests were conducted by the investigator with at least a 1-hour time interval between the applications of both, in private and in quiet surroundings, after thorough explanation to the participant of the aim of the study, the specifics of the test to be done, and after preliminary signed informed consent for the participation in a scientific study. Hachinski Ischemic Score was used to assess the risk for vascular origin of dementia $[6,16]$. This is a simple clinical tool used to discriminate between types of dementia (primary degenerative dementia: $<4$ points, vascular or multi-infarct dementia: $>7$ points, mixed type dementia: 4-7 points). Some studies have assessed its validity, and it demonstrated good sensitivity and specificity in defining degenerative or vascular dementia. But its use in the early stages of cognitive impairment has not been widely tested.

\section{Study Population}

In everyday practice, it is almost impossible to find patients with isolated cardiovascular risk factors, especially when considering the elderly population. There is a clear tendency for cardiovascular risk factors to cumulate with the leading role of arterial hypertension. The study population consists of 325 patients from all over Bulgaria, all of whom had arterial hypertension of variable duration. All patients received medical therapy for BP control, and the majority of them were on combination therapy. Inclusion criteria were patients with known and treated hypertension. In the presence of atrial fibrillation optimal anticoagulation at the time of hospitalization was a prerequisite. Exclusion criteria were: (1) diagnosed Alzheimer's disease, Parkinson's disease, and other forms of dementia; (2) acute de-compensated or chronic renal failure stage II and III, and end-stage renal disease; (3) liver failure; (4) decompensated hypothyroidism or hyperthyroidism; (5) poorly controlled diabetes mellitus; (6) history of extracorporeal circulation; (7) history of coma, serious head trauma, epilepsy; (8) antidepressants/antipsychotics, and (9) atrial fibrillation with poor anticoagulation.

\section{Characteristics of the Study Population}

The study population (table 1) consisted of 119 (36.6\%) males and 206 (63.4\%) females, $52(16 \%)$ had atrial fibrillation on anticoagulation therapy, 89 (27.4\%) had dyslipidemia, 160 $(49.2 \%)$ were on treatment for diabetes mellitus or impaired glucose tolerance, 41 (38.2\%) had fully compensated renal disease (creatinine clearance $>60 \mathrm{ml} / \mathrm{min}$ ), and $30(9.2 \%)$ had treated and compensated thyroid dysfunction.

\section{Data Analysis and Statistics}

The threshold for home-measured BP was chosen in accordance with the ESC and ESH recommendations for home-measured $\mathrm{BP}$, namely $135 / 85 \mathrm{~mm} \mathrm{Hg}$ independently of any con- 
Fig. 1. Differences in the neuropsychological test results between the groups with optimal and suboptimal BP control. HIS = Hachinski Ischemic Score.

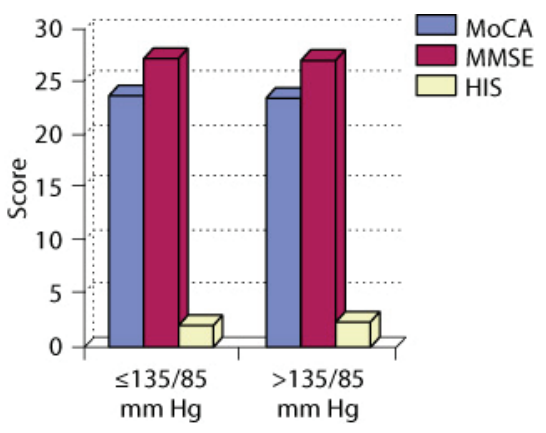

Table 1. Characteristics of the study population

$\begin{array}{lr}\text { Subjects, } \mathrm{n} & 325 \\ \text { Age, years } & 66.12 \pm 10.10 \\ \text { Hypertension history, years } & 13.26 \pm 11.26 \\ \text { Systolic BP, mm Hg } & 141.45 \pm 18.45 \\ \text { Diastolic BP, mm Hg } & 85.51 \pm 11.17\end{array}$

comitant diabetes and renal disease [17]. SPSS was used for data analysis. We found that the self-reported rate of suboptimal BP control in treated hypertensive patients was $65.62 \%$. MannWhitney nonparametric statistical analysis was used to assess if there is any statistically significant difference $(\alpha<0.05)$ between the mean values of the neuropsychological test results.

Comparison of the mean values of neuropsychological test results in patients with optimal and suboptimal BP control without considering age (fig. 1) showed that there was no significant difference between neuropsychological test results of the two groups. In the group with suboptimal control the mean value for MMSE was $27.27( \pm 2.78)$ points, for MoCA 23.67 ( \pm 3.76$)$ points, and for Hachinski $2.44( \pm 1.78)$ points. In the group with optimal control the values were MMSE 27.40 ( \pm 2.856$)$ points, MoCA 24.00 ( \pm 4.05$)$ points, and Hachinski $1.96( \pm 1.53)$ points. This means that we could not draw conclusions for the group as a whole without considering other factors. If we considered only the patients without atrial fibrillation, the results were corresponding, namely there was no significant difference between the neuropsychological test results between the groups with suboptimal and optimal BP control and sinus rhythm.

Analysis showed significantly $(\alpha<0.5)$ lower neuropsychological test results in patients with atrial fibrillation (MoCA 23.67 points, MMSE 27.27 points, and Hachinski 2.44 points) compared with those without atrial fibrillation (MoCA 24.0 points, MMSE 27.4 points, and Hachinski 1.96 points; fig. 2).

Additionally, we studied the population by age. We found that there is a clear tendency for the neuropsychological test results, respectively cognitive impairment, to decline with age for patients with suboptimal BP control (fig. 3). This tendency is obscured above the age of 80 years. Analogous results could be observed for the whole study population as well as for the selected patients without atrial fibrillation.

Regression analysis shows that there is a medium-strength positive correlation between age and BP values and neuropsychological test results (table 2). This means that, with advancing age, too low a BP may not be as positive as it was considered previously. The question about the precise threshold value both for age and for BP remains still an open issue of debate. 
Fig. 2. Comparison of neuropsychological test results between the groups of hypertensive patients with atrial fibrillation (afib) and hypertensive patients without atrial fibrillation. HIS = Hachinski Ischemic Score.

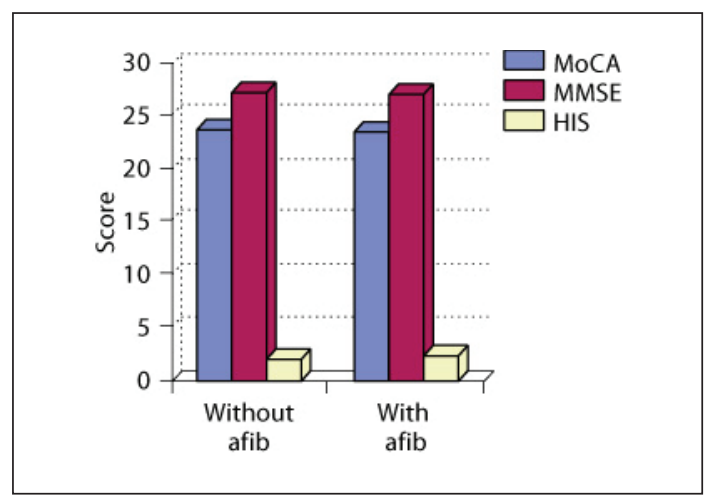

Fig. 3. Neuropsychological test results by age intervals in the whole study population. HIS = Hachin-

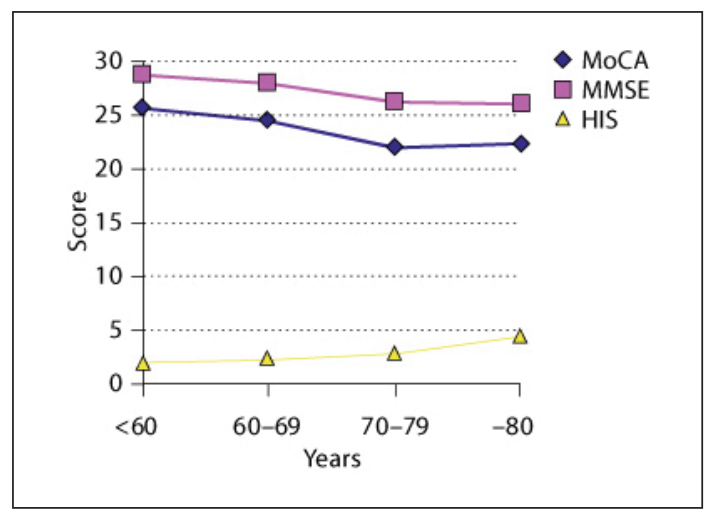
ski Ischemic Score.

Table 2. Strength and direction of the correlation between neuropsychological test results and $\mathrm{BP}$ values in combination with age

\begin{tabular}{lll}
\hline Factors & $\begin{array}{l}\text { MoCA correlation } \\
\text { coefficient }\end{array}$ & $\begin{array}{l}\text { MMSE correlation } \\
\text { coefficient }\end{array}$ \\
\hline Age/SBP & 0.362 & 0.360 \\
Age/DBP & 0.361 & 0.368 \\
\hline
\end{tabular}

We also found that there is a significant $(\alpha<0.5)$ difference between the mean values of the neuropsychological test results of the groups with concomitant and without concomitant risk factors in the patients with suboptimal BP control (fig. 4). Analogous results can be observed for the selected patients without atrial fibrillation. The risk factors that showed a significant correlation with cognitive impairment were diabetes mellitus or impaired glucose tolerance and history of renal disease. Patients with diabetes and/or a history of renal disease had a significantly $(\alpha<0.5)$ poorer presentation on neuropsychological tests (MoCA 22.47 points, MMSE 26.85 points, and Hachinski 2.85 points) than their healthier counterparts without these risk factors (MoCA 24.41 points, MMSE 27.74 points, and Hachinski 2.09 points). Other factors, such as compensated thyroid dysfunction, seemed to play a minor role in cognitive impairment as long as they were fully compensated.

\section{Conclusion}

Hypertensive patients with atrial fibrillation are at a higher risk for cognitive impairment despite optimal coagulation therapy at the time of testing. There is a correlation be- 
Fig. 4. Comparison of neuropsychological test results between the groups of hypertensive patients with risk factors and without. HIS = Hachinski Ischemic Score.

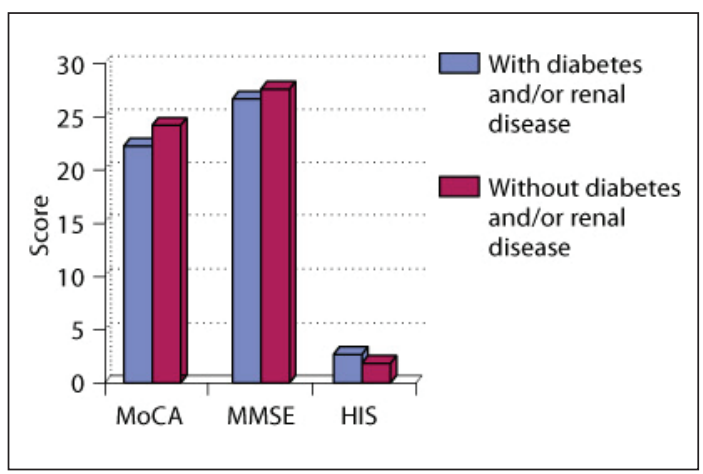

tween suboptimal home-measured BP and early stages of cognitive impairment. The result is age dependent. Patients with concomitant risk factors such as diabetes and renal disease are at an increased risk for cognitive impairment. Cognitive decline is dependent on multiple factors. Suboptimal BP control in the very elderly is not correlated with an added risk of cognitive impairment above the age-related risk.

\section{Discussion}

A home-measured BP that is not in the recommended range is an important vascular risk factor for cognitive impairment in the young, but not in the elderly. It is important to consider both, age and hypertension, especially when screening for early cognitive impairment. Most of the neuropsychological tests do not account for age and education. Our team suggests that age be included in neuropsychological tests. Recommended BP should vary according to age. BP acts in combination with other factors on cognitive impairment. Some treatments may have a protective role against cognitive impairment in certain risk groups, although further studies are needed. The use of highly specific and sensitive neuropsychological tests for the early diagnosis of cognitive impairment in patients with home-measured $\mathrm{BP}$ above the recommended range is an effective screening tool in the everyday practice.

\section{Future Directions}

The team is currently working on: (1) the development of a time-sparing and easy-toadminister score system for cognitive impairment risk assessment in everyday practice; (2) finding BP thresholds that are age and risk factor specific, and (3) elucidating the protective effect of hypertensive treatment of early cognitive impairment according to the cardiovascular risk profile.

\section{Limitations}

A major limitation of our study is the lack of neuroimaging modalities - at best MRI imaging. We recognize the need to correlate the clinical findings with a specific brain localization. Along with this, neuroimaging might provide us with important clues on the specific type of brain lesions, neuropsychological profile, and differential diagnosis of mild cognitive impairment/vascular cognitive impairment. The large number of patients included in the study and the relatively high price of MRI testing were the main impediments to acquiring the needed neuroimaging. Along with this, the focus of our work is on primary detection (possibly during prophylactic medical visits) and initial screening for cognitive impairment as well as defining high-risk groups, which will benefit most from such a screening. 


\section{References}

1 World Health Organization: The World Health Report. www.who.int/whr/1997/media_centre/ executive_summaryl/en/index.html.

2 Cognitive Impairment in the Elderly - Recognition, Diagnosis and Management. British Columbia Ministry of Health. Revised January 30, 2008.

-3 Gauthier S, Reisberg B, Zaudig M, Petersen RC, Ritchie K, Broich K, Belleville S, et al: International Psychogeriatric Association Expert Conference on mild cognitive impairment. Mild cognitive impairment. Lancet 2006;367:1262-1270.

-4 Huang J, Meyer J, Zhang Z, Wei J, Hong X, Wang J, Wen H, Wu W, et al: Progression of mild cognitive impairment to Alzheimer's or vascular dementia versus normative aging among elderly Chinese. Curr Alzheimer Res 2005;2:571-578.

-5 Wiederkehr S, Laurin D, Simard M, Verrault M, Lindsay J: Vascular risk factors and cognitive functions in nondemented elderly. J Geriatr Psychiatry Neurol 2009;22:196-206.

-6 Hachinski V, Iadecola C, Petersen RC, Breteler MM, Nyenhuis DL, Black SE, Powers WJ, et al: National Institute of Neurological Disorders and Stroke-Canadian Stroke Network vascular cognitive impairment harmonization standards. Stroke 2006;37:2220-2241.

-7 Vasan R, Beiser A, Seshadri S, Larson M, Kannel W, D’Agostino R, Levy D: Residual lifetime risk for developing hypertension in middle-aged women and men: The Framingham Heart Study. JAMA 2002;287:1003-1010.

-8 Grassi G, Cifkova R, Laurent S, Narkiewicz K, Redon J, Farsang C, Viigimaa M, et al: Blood pressure control and cardiovascular risk profile in hypertensive patients from central and eastern European countries: results of the BP-CARE study. Eur Heart J 2011;32:218-225.

-9 Cacciatore F, Abete P, de Santis D, Longobardi G, Ferrara N, Rengo F: Mortality and blood pressure in elderly people with and without cognitive impairment. Gerontology 2005;51:53-61.

10 Mancia G: Effects of intensive blood pressure control in the management of patients with type 2 diabetes mellitus in the Action to Control Cardiovascular Risk in Diabetes (ACCORD) Trial. Circulation 2010;122:847-849.

-11 Obisesan TO, Obisesan OA, Martins S, Alamgir L, Bond V, Maxwell C, Gillum RF: High blood pressure, hypertension, and high pulse pressure are associated with poorer cognitive function in persons aged 60 and older: the Third National Health and Nutrition Examination Survey. J Am Geriatr Soc 2008;56:501-509.

-12 Parati G, Stergiou GS, Asmar R, Bilo G, de Leeuw P, Imai Y, Kario K, et al; ESH Working Group on Blood Pressure Monitoring: European Society of Hypertension practice guidelines for home blood pressure monitoring. J Hum Hypertens 2010;24:779-785.

-13 O’Brien E, Asmar R, Beilin L, Imai Y, Mallion JM, Mancia G, Mengden TH, et al; European Society of Hypertension Working Group on Blood Pressure Monitoring: Practice guidelines of the European Society of Hypertension for clinic, ambulatory and self blood pressure measurement. J Hypertens 2005;23:697-701.

14 Nasreddine ZS, Phillips NA, Bédirian V, Charbonneau S, Whitehead V, Collin I, Cummings JL, Chertkow H: The Montreal Cognitive Assessment, MoCA: a brief screening tool for mild cognitive impairment. J Am Geriatr Soc 2005;53:695-699.

-15 Smith T, Gildeh N, Holmes C: The Montreal Cognitive Assessment: validity and utility in a memory clinic setting. Can J Psychiatry 2007;52:329-332.

-16 Mölsä PK, Paljärvi L, Rinne JO, Rinne UK, Säkö E: Validity of clinical diagnosis in dementia: a prospective clinicopathological study. J Neurol Neurosurg Psychiatry 1985;48:1085-1090.

-17 Mancia G, Laurent S, Agabiti-Rosei E, Ambrosioni E, Burnier M, Caulfield MJ, Cifkova R, et al; European Society of Hypertension: Reappraisal of European guidelines on hypertension management: a European Society of Hypertension Task Force document. J Hypertens 2009;27:2121-2158. 